\title{
Conception of a New Recoil Proton Telescope for Real-Time Neutron Spectrometry in Proton-Therapy
}

\author{
Rodolphe Combe, Nicolas Arbor, Ziad el Bitar, Stéphane Higueret, Daniel Husson \\ Université de Strasbourg \\ CNRS, IPHC UMR 7178 \\ F-67000 Strasbourg, France \\ Email: rodolphe.combe@iphc.cnrs.fr
}

\begin{abstract}
Neutrons are the main type of secondary particles emitted in proton-therapy. Because of the risk of secondary cancer and other late occurring effects, the neutron dose should be included in the out-of-field dose calculations. A neutron spectrometer has to be used to take into account the energy dependence of the neutron radiological weighting factor. Due to its high dependence on various parameters of the irradiation (beam, accelerator, patient), the neutron spectrum should be measured independently for each treatment.

The current reference method for the measurement of the neutron energy, the Bonner Sphere System, consists of several homogeneous polyethylene spheres with increasing diameters equipped with a proportional counter. It provides a highresolution reconstruction of the neutron spectrum but requires a time-consuming work of signal deconvolution. New neutron spectrometers are being developed, but the main experimental limitation remains the high neutron flux in proton therapy treatment rooms. A new model of a real-time neutron spectrometer, based on a Recoil Proton Telescope technology, has been developed at the IPHC. It enables a real-time high-rate reconstruction of the neutron spectrum from the measurement of the recoil proton trajectory and energy. A new fast-readout microelectronic integrated sensor, called FastPixN, has been developed for this specific purpose.

A first prototype, able to detect neutrons between 5 and $20 \mathrm{MeV}$, has already been validated for metrology with the AMANDE facility at Cadarache. The geometry of the new Recoil Proton Telescope has been optimized via extensive Geant 4 Monte Carlo simulations. Uncertainty sources have been carefully studied in order to improve simultaneously efficiency and energy resolution, and solutions have been found to suppress the various expected backgrounds. We are currently upgrading the prototype for secondary neutron detection in proton therapy applications.
\end{abstract}

\section{INTRODUCTION}

\section{A. Secondary neutrons in proton-therapy}

During a proton-therapy treatment, a lot of secondary neutrons are produced by the interaction of the proton beam with the patient (internal neutrons), and with the accelerator (external neutrons) for some treatments, via ${ }_{Z}^{A} X(p, n)_{Z+1}^{A} Y$ reactions. Due to their high radiological weighting factor, the neutron dose should be monitored. It can grow up to $500 \mathrm{mGy}$ over a full treatment. It has been shown in [1] that this dose can vary greatly depending on the treatment methods and other parameters. As the biological effect of neutrons is dependent on their energy [2], a neutron spectrometry is mandatory for a precise estimation of the neutron equivalent dose. The fast neutrons being the greatest contributors to the neutron dose, our approach is to focus on this energy range while other systems are developed for measuring thermal neutrons.

The neutron spectrum depends among others on:

- the energy of the protons, which mainly changes the fluence and shifts the endpoint of the spectrum to the energy of the beam [3][4]

- the angle to the proton beam, which changes the shape of the fast neutron spectrum [5][6][7][8][9]

- the distance to isocentre, which changes not only the fluence but also the shape of the spectrum [10][11][12]

Because of that, one spectrum can only be identified to one treatment. Scarce experimental data coupled to extrapolation via Monte Carlo simulation is currently used to estimate the dose, but this leads to large discrepancies between the simulation and experimental data.

\section{B. Neutron spectrometry}

The current reference spectrometer for neutrons is the Bonner Sphere System, which has been extensively used and studied in literature. It is composed of several spheres of homogeneous polyethylene of increasing diameters, equipped with a ${ }^{3} \mathrm{He}$ proportional counter in their center. This system allows a precise reconstruction of the spectrum over a large energy range. However, it requires a complex procedure of deconvolution and both the measurement and the deconvolution are time-consuming, which excludes a real-time measurement. More problematic for this application, it is cumbersome and it has been proven in [13] to be limited to a fluence of $4 \times 10^{4} \mathrm{n} / \mathrm{cm}^{2} / \mathrm{s}$, which is two orders of magnitudes below the $6 \times 10^{6} \mathrm{n} / \mathrm{cm}^{2} / \mathrm{s}$ that can be found in treatment rooms [1]. Because of that, the Bonner Sphere System cannot be used in treatment rooms, and is far from being optimal for multiparameter studies.

Other neutron spectrometers are currently being developed as the MONDO project [14][15] or the capture-gated detector FANS-2 [16]. The first project aims at measuring the double elastic scattering of fast neutrons by the means of arrays of scintillating fibers. It will allow to measure neutrons between 20 and $400 \mathrm{MeV}$ with a size of $10 \times 10 \times 20 \mathrm{~cm}^{3}$. The second project combines plastic scintillators and ${ }^{3} \mathrm{He}$ counters to measure neutrons between a few $\mathrm{MeV}$ and $1 \mathrm{GeV}$. However, it is $1.15 \mathrm{~m}$ long, can only measure $200 \mathrm{n} / \mathrm{cm}^{2} / \mathrm{s}$ and requires ${ }^{3} \mathrm{He}$, which is both expensive and requires high-voltage. 


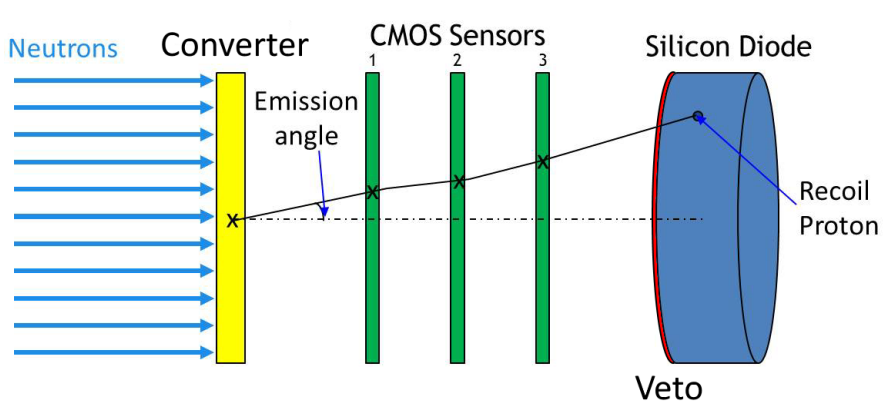

Fig. 1. Scheme of the Recoil Proton Telescope

Our prototype is a new real-time neutron spectrometer based on a Recoil Proton Telescope that has been developed at IPHC. To go beyond existing so-called "zero-degree" telescopes, we choose to reconstruct the full track of recoil protons, inside an opening angle of $41^{\circ}$ (95\% C.L.). The CMOS pixels Recoil Proton Telescope allows a real-time high-rate $(100 \mathrm{kHz})$ of the fast neutron spectrum within a limited size $(10 \times 15 \times$ $20 \mathrm{~cm}^{3}$ ). Extensive Monte Carlo Geant4 [17] simulations have been carried out to characterize precisely the performances of this device.

\section{Recoil Proton Telescope}

\section{A. Principle}

Fast neutrons $\left(E_{n}>1 \mathrm{MeV}\right)$ are elastically scattered on a hydrogenated converter (polyethylene). The energy $E_{p}$ and emission angle $\theta$ of each recoil proton is directly related to the incident neutron energy $E_{n}$ :

$$
E_{n}=\frac{E_{p}}{\cos ^{2} \theta}
$$

The emission angle will be measured using three CMOS sensors and the energy of the proton using a silicon diode. This will allow to measure the neutron energy in real-time with a rate a $100 \mathrm{kHz}$.

The full scheme of the Recoil Proton Telescope is presented on figure 1.

\section{B. Trajectography}

To reconstruct the trajectory, a monolithic active pixels CMOS sensor named FastPixN [18] is used. It is made of $128 \times 128$ pixels of $50 \mu \mathrm{m}$ pitch. It has been thinned down to $50 \mu \mathrm{m}$ to minimize the energy loss inside the Recoil Proton Telescope. With 4-bit column-ADC, the acquisition rate of $100 \mathrm{kHz}$ allows to measure up to $10^{9} \mathrm{n} / \mathrm{cm}^{2} / \mathrm{s}$.

The uniformity of these sensors has been tested at the AIFIRA platform (CENBG Bordeaux). Despite the difficulty to calibrate CMOS sensors, due to their charge collection through thermal diffusion, a good calibration curve was achieved up to $20 \mathrm{MeV}$. This allows to use the energy deposited in the FastPixN as a criterion for the analysis, to constrain the direction of the proton.

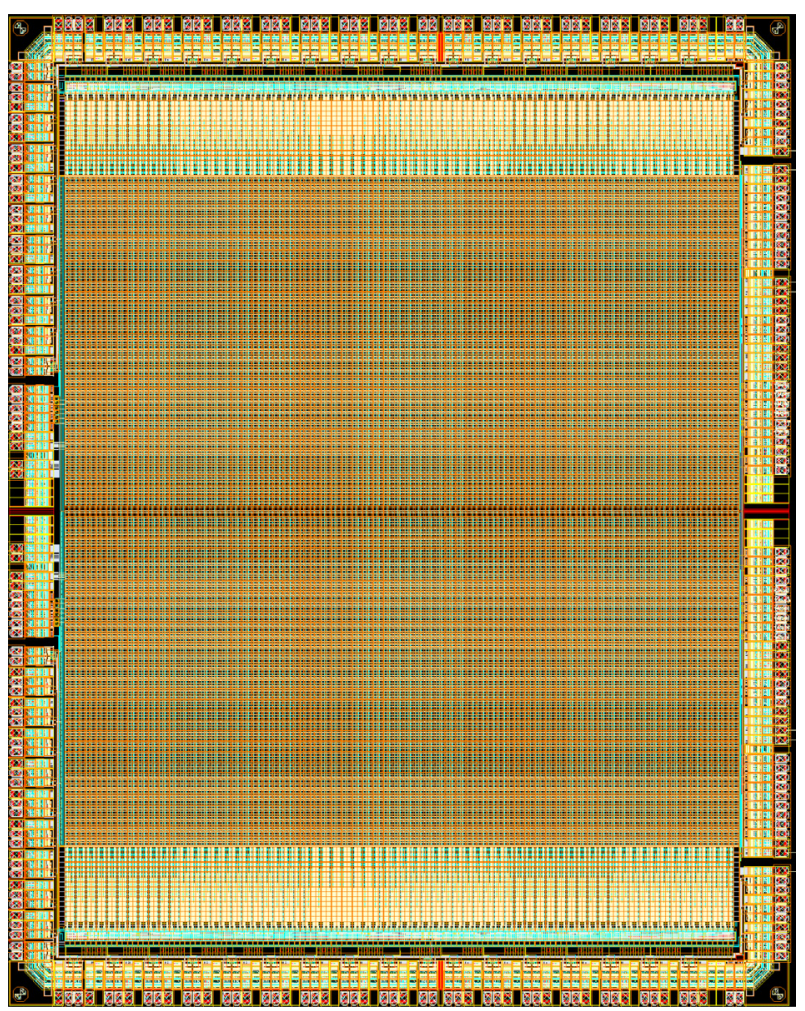

Fig. 2. View of the FastPixN sensor

\section{Reconstruction of the neutron energy}

To measure the proton energy, the Recoil Proton Telescope uses a silicon diode read by a 13-bit ADC. The thickness of the diode has been set as an optimum between charge collection and proton stopping power. This 3-mm-thick diode can measure protons with energies up to $20 \mathrm{MeV}$. The proton initial energy is estimated as the sum of the energy deposited in the diode and the estimated mean energy loss in the Recoil Proton Telescope, which has been calculated using a Monte Carlo Geant4 simulation for each deposited energy. This mean energy loss is corrected by the estimated track length, determined by the measurement of the angle between the three sensor hits.

\section{First prototype}

A first prototype of Recoil Proton Telescope has been designed for the Laboratory of Neutron Metrology and Dosimetry (LMDN) of Institute of Radio-protection and Nuclear safety (IRSN) Cadarache (figure 3). It provides a good reconstruction of mono- and poly-energetic neutrons spectrum between 5 and $20 \mathrm{MeV}$. The requirements for this device were a $5 \%$ resolution on $E_{n}$, low background and high efficiency for moderate fluences $\left(<6000 \mathrm{n} / \mathrm{cm}^{2} / \mathrm{s}\right)$.

\section{MEASUREMENT OF THE PROTON-THERAPY NEUTRON SPECTRUM}

To measure the proton-therapy neutron spectrum, two different sensors will be used. The first one, called AlphaRad [19], 


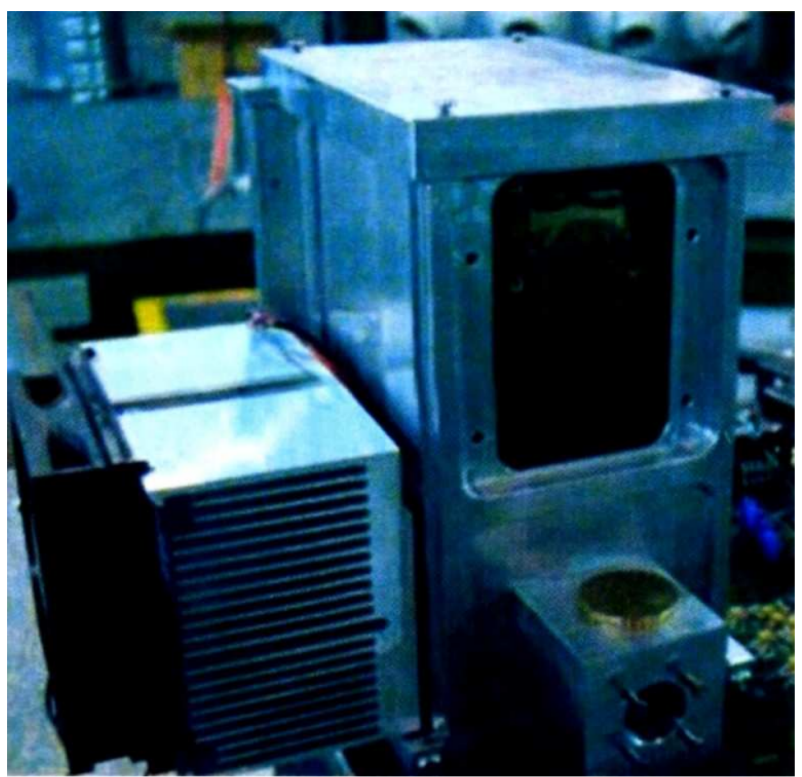

Fig. 3. View of the first prototype

will count thermal and fast neutron under $5 \mathrm{MeV}$. The Recoil Proton Telescope will be used to reconstruct the fast neutron spectrum over $5 \mathrm{MeV}$. These experimental measurements will be used, in a first time, to cross-check the various nuclear models used for the dose calculation by the Treatment Planning Softwares and the Monte Carlo simulation codes. Since the telescope has been first optimized for the metrological application at IRSN, we need to use this device over an extended energy range, first up to $30 \mathrm{MeV}$, to address the survey of the neutron production in proton-therapy. As has been shown in literature, this energy range $([5,30] \mathrm{MeV})$ is interesting, especially at high angle and for low energy irradiation such as ocular treatments.

\section{ANALYSIS}

\section{A. Uncertainty sources}

To improve the reconstruction of the neutron energy, an ideal simulation of the Recoil Proton Telescope, with protons created randomly inside the converter and full knowledge about the trajectory and the energy deposits, was generated. This simulation allowed to study what are the critical parameters for the energy resolution and which ones can eventually be modified.

For example, for $10-\mathrm{MeV}$ protons with a $50-\mu \mathrm{m}$-thick converter, the uncertainty $(1 \sigma)$ over the reconstruction of the proton energy is found to be of $0.86 \pm 0.01 \%$ (figure 4 ).

The largest uncertainty source is found to be the inability to determine the position of creation of the protons inside the converter through the elastic $\mathrm{H}(\mathrm{n}, \mathrm{n}) \mathrm{p}$ reaction. It creates an uncertainty of $0.71 \pm 0.01 \%$ on the proton energy. The other sources of uncertainty are the energetic and angular straggling inside the CMOS, which contribute to $0.50 \pm 0.01 \%$, and in the air, which contribute to $0.13 \pm 0.01 \%$. Concerning the angular resolution, the uncertainty on $\cos \theta$ is found to be $0.30 \pm 0.01 \%$.

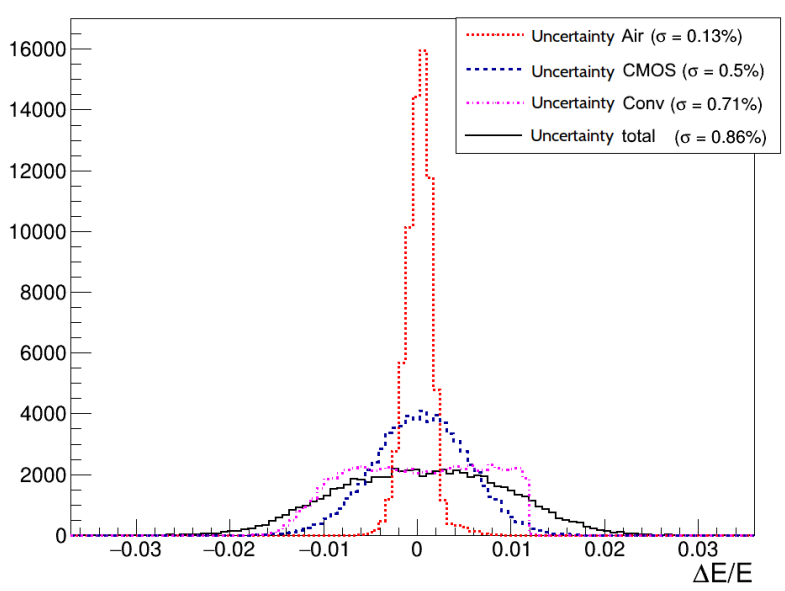

Fig. 4. Contribution of the various uncertainty sources to the total uncertainty (dotted) and total uncertainty (solid) in the reconstruction of the proton energy (Geant4 simulation)

\section{B. Background suppression}

1) Coherent background sources:

As the conversion efficiency of a neutron into a proton is of only $10^{-3}$ per millimeter of polyethylene, a high background coming from direct neutron hits in the diode is expected. To prevent the contamination of the signal by these hits, a $65-\mu \mathrm{m}$ veto diode was installed right before the 3-mm-thick diode. A collected track is then validated only if it has one hit in each CMOS plus one hit in the veto.

The high reading frequency of the FastPixN allows a high reduction of the number of neutrons, and therefore protons, per frame, reducing by the way the background. Moreover, an extensive analysis of the geometric and energetic distribution of the events allows to suppress tracks which do not correspond to signal events.

Several other background sources are expected, each of them requesting a dedicated cut, listed thereafter:

- Proton created in the diode: a $\mathrm{Si}$ nucleus of the diode scattering inelastically with a neutron can produce one or more protons through $\mathrm{Si}(\mathrm{n}, \mathrm{sp})$ reactions. A proton flying back through the device would produce a track similar to a signal event. To suppress these events, an increasing energy deposit inside successive CMOS is requested, in accordance with the Bethe-Bloch model.

- Hydrogen isotopes: consecutive to a neutron capture on hydrogen, a deuteron or triton can be ejected, mimicking the signal of a lower energy proton. These events are suppressed by requesting the energy deposited in the veto and in the diode to correspond to those of a proton in accordance with the Bethe-Bloch model.

- Proton created out of the converter: during the inelastic scattering of a neutron, a proton can be produced, which will then generate a track correspond- 
ing to a lower energy neutron. To suppress these events, the trajectory of the proton is extrapolated towards the converter.

- Proton escaping the diode: a proton arriving at the periphery of the diode with a certain angle can leave it without depositing its full energy. To suppress these events, it is verified that the proton can't escape the diode by extrapolating its trajectory towards the diode.

2) Fortuitous coincidence:

Because of the high neutron flux in proton-therapy treatment rooms and despite the high-rate capability of the Recoil Proton Telescope, fortuitous coincidences are a major background source. To study this source, PCB boards as well as the metallic box, that are a major source of secondaries, were added to the simulation. Moreover, the Recoil Proton Telescope was irradiated in simulation within a solid angle covering the whole box. This allowed to study extensively the effect of fortuitous coincidences.

There are two background sources related to fortuitous coincidences. The first one is the coincidence of a direct neutron hit in the diode with a partial track, corresponding to a proton stopped in the last CMOS or right after. The second one is the coincidence of a neutron hit with a signal event. These two backgrounds are easily suppressed by requesting a hit in the veto for the first one and a correlation between the energy deposited in the veto and the diode for the second one corresponding to the Bethe-Bloch model.

3) Cuts:

Hereafter are listed the applied cuts:

a) Increasing energy deposit and Bragg peak:

- From the Bethe-Bloch model, (in this energy range) a particle losing its energy has an increasing energy deposit along the track. This means that recoil protons should deposit more and more energy along their trajectory in the Recoil Proton Telescope. Therefore, the energy deposited in the CMOS sensors should be increasing for a signal event. This is softened by the poor energy resolution of the CMOS sensors (4 bits) and by the energetic straggling.

- At the end of its track, a proton will deposit a lot of energy over a very short distance: this is the so-called "Bragg peak". A proton whose Bragg peak is observed in a CMOS cannot therefore reach the diode.

The applied criterion is then an increasing energy deposit to the bit level and a lack of the Bragg peak.

$$
\triangle E_{F P N} \in[-1 \text { bit; }+2 \text { bits }]
$$

b) Correlation Veto-Diode:

The Bethe-Bloch model also allows to establish a correlation between the energy deposited in the
TABLE I

EFFICIENCY OF THE CUTS

\begin{tabular}{|c||c|c|c|c|}
\hline Cut & Efficiency & Purity & Resolution & S/(S+B) \\
\hline Raw data & & & $31.3 \pm 0.5 \%$ & $24.8 \pm 0.7 \%$ \\
\hline$\Delta F P N(2)$ & $5.7 \pm 0.7 \%$ & $99.7 \pm 0.1 \%$ & $30.8 \pm 0.5 \%$ & $23.8 \pm 0.7 \%$ \\
\hline$\Delta$ Veto (3) & $87.1 \pm 1 \%$ & $75.1 \pm 0.8 \%$ & $19.3 \pm 0.4 \%$ & $5.5 \pm 0.5 \%$ \\
\hline R0 (6) \& R4 (7) & $6.6 \pm 0.8 \%$ & $97.2 \pm 0.3 \%$ & $31.0 \pm 0.5 \%$ & $24.1 \pm 0.7 \%$ \\
\hline All cuts & $89.8 \pm 0.9 \%$ & $73.2 \pm 0.8 \%$ & $17.9 \pm 0.4 \%$ & $4.4 \pm 0.4 \%$ \\
\hline
\end{tabular}

veto and in the diode. The energy deposited in the diode $E_{\text {meas }}^{\text {diode }}$ allows to estimate the energy $E_{t h}^{\text {veto }}$ that should be deposited in the veto by a proton depositing $E_{\text {meas }}^{\text {diode }}$ in the diode. By comparing with the energy deposited in the veto $E_{\text {meas }}^{\text {veto }}$, a criterion on the validity of the track is obtained. Because of the energetic straggling and after optimization, this criterion is expressed as an upper limit:

$$
\frac{E_{\text {th }}^{\text {veto }}-E_{\text {meas }}^{\text {veto }}}{E_{\text {meas }}^{\text {veto }}}<9 \%
$$

c) Extrapolation of the track:

To verify that the proton emerges from the converter and ends its track inside the diode, its track can be extrapolated in the two directions to cancel background events. With $\left(X_{1}, Y_{1}\right)$ to $\left(X_{3}, Y_{3}\right)$ the coordinates of the hits in the CMOS and after optimization, the coordinates of the proton inside the converter and the diode can be extrapolated to:

$$
\begin{array}{r}
\left(X_{0}, Y_{0}\right) \approx\left(2 * X_{1}-X_{2}, 2 * Y_{1}-Y_{2}\right) \\
\left(X_{4}, Y_{4}\right) \approx\left(2 * X_{3}-X_{2}, 2 * Y_{3}-Y_{2}\right) \\
R_{0}=\sqrt{X_{0}^{2}+Y_{0}^{2}}<5.1 \mu \mathrm{m} \\
R_{4}=\sqrt{X_{4}^{2}+Y_{4}^{2}}<6.0 \mu \mathrm{m}
\end{array}
$$

Table I presents the efficiency, purity, resolution on $E_{n}$ and background level of the various cuts.

\section{STUdy OF THE GEOMETRY}

\section{A. Material of the box}

According to the literature [20], our aluminum box has a high cross section of secondary production via $\mathrm{Al}(\mathrm{n}, \mathrm{p})$ and $\mathrm{Al}(\mathrm{n}, \mathrm{n}+\mathrm{p})$ reactions of around $100 \mathrm{mb}$. Other materials frequently used near accelerators, such as tungsten and lead, have a secondary production cross-section 10 times lower but an overall neutron cross-section 5 times higher. After simulation (Table II), only a small benefit of using tungsten is found. It is therefore chosen to keep aluminum which is easier to process.

\section{B. Distance to the diode}

As the third PCB board and the box are two important sources of secondaries, the diode, which lies between them, should be carefully placed. After simulation (figure 5), the efficiency is found to decrease with the distance between the 
TABLE II

EFFECT OF THE BOX MATERIAL ON RPT PERFORMANCES

\begin{tabular}{|c||c|c|}
\hline Material & $\mathrm{Al}$ & $\mathrm{W}$ \\
\hline Efficiency $\left(\times 10^{-5}\right)$ & $2.4 \pm 0.05$ & $2.22 \pm 0.04$ \\
\hline $\mathrm{B} /(\mathrm{S}+\mathrm{B})$ & $7.7 \pm 0.7 \%$ & $12.8 \pm 0.7 \%$ \\
\hline Resolution on $E_{n}$ & $20.2 \pm 0.5 \%$ & $18.7 \pm 0.4 \%$ \\
\hline
\end{tabular}
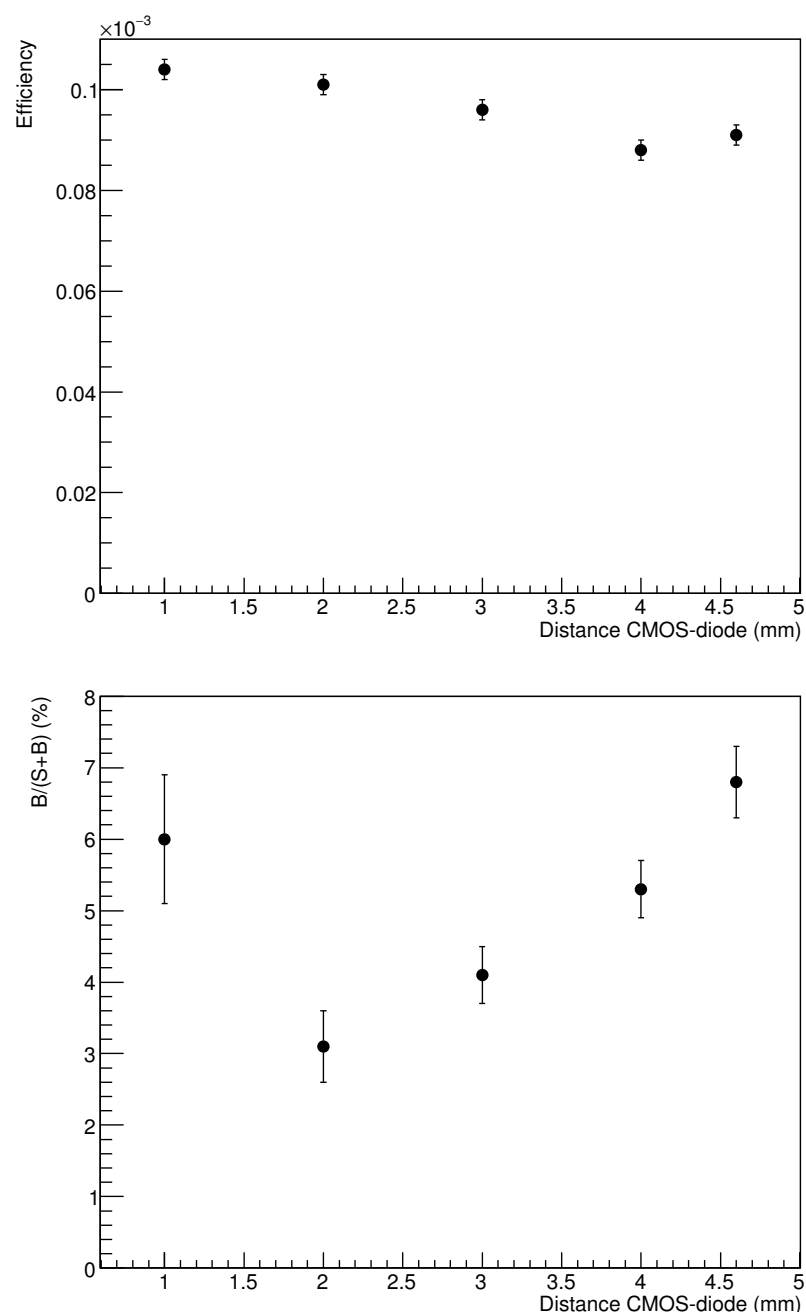

Fig. 5. Fiducial measurement efficiency (right) and background level (left) for a proton therapy spectrum (Geant4 simulation)

third CMOS and the diode. This is due to the decrease of the aperture angle. An optimum for the background level is also found for a distance of $2 \mathrm{~mm}$. If the diode is too close to the third CMOS, it will receive the high-angle proton that should escape the diode. If it is too close to the box, it will detect the secondaries coming from inelastic events in the box. An optimum is then found for a $2 \mathrm{~mm}$ distance. The mechanical realization of such a small distance is under investigation.

\section{Converter thickness}

Because of the high-energy of neutrons in proton-therapy treatment rooms, the $\mathrm{H}(\mathrm{n}, \mathrm{n}) \mathrm{p}$ cross-section is low and the
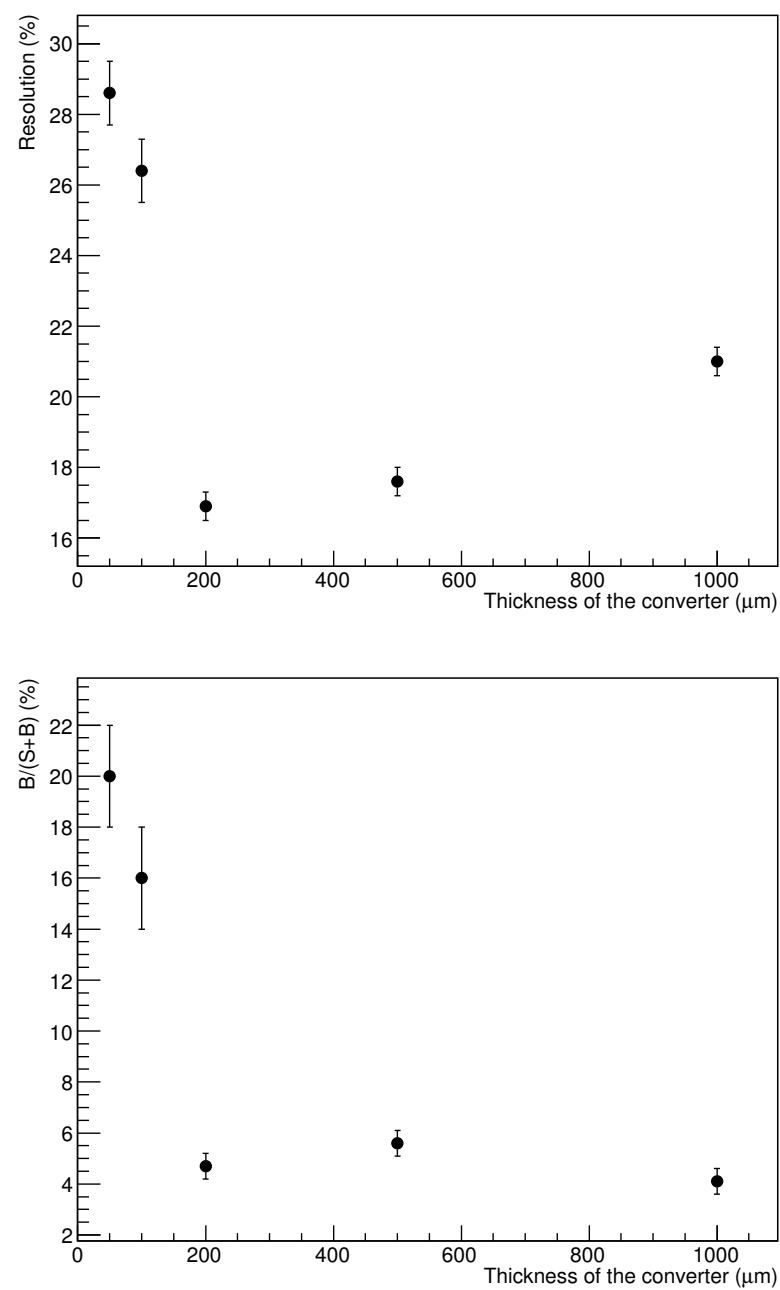

Fig. 6. Resolution on the neutron energy (right) and background level (left) for a proton therapy spectrum as a function of the distance between the diode and the third CMOS (Geant4 simulation)

secondaries production cross-section is high. This creates a high background level that needs to be compensated by a high production efficiency. However, to achieve this high efficiency, the converter needs to be thickened and it has been seen in section IV-A that this will degrade the resolution on $E_{p}$ and therefore on $E_{n}$.

An optimum for the resolution is found between 200 and $500 \mu \mathrm{m}$ of polyethylene (figure 6). If the converter is too thin, the signal-over-noise ratio is low, which can also be seen through the background level, and if it is too thick, the resolution on $E_{p}$ starts to be degraded.

\section{FIRST RESULTS UP TO $30 \mathrm{MEV}$}

For a proton-therapy spectrum corresponding to an irradiation of the head with a $178 \mathrm{MeV}$ proton beam [13], a reconstruction of the neutron spectrum is obtained between 5 to $30 \mathrm{MeV}$ with a $16.3 \pm 0.3 \%$ resolution. As can be seen in figure 7 , the main reason for this resolution is the high level 


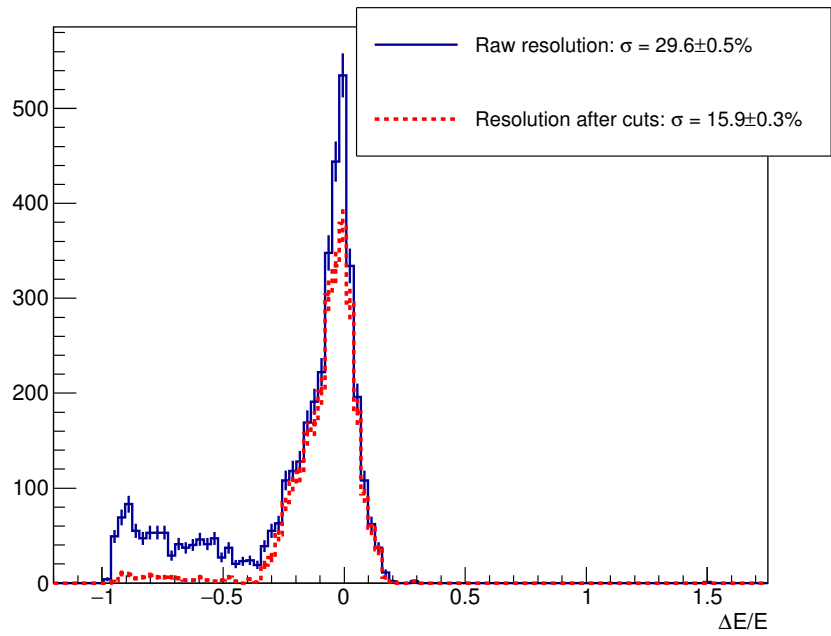

Fig. 7. Raw resolution (solid) and after-cut resolution (dotted) on $E_{n}$ for a proton therapy spectrum (Geant4 simulation)

of background that can be seen in the tail corresponding to a $\Delta E / E$ smaller than -0.4 . This resolution should be sufficient to benchmark nuclear models of neutron production in protontherapy.

\section{CONCLUSION AND PROSPECTIVE}

The possibility of using a new Recoil Proton Telescope as a mean to measure secondary neutrons spectrum in protontherapy treatment room has been proven. A first prototype is currently being built and should be able to measure neutrons between 5 and $30 \mathrm{MeV}$.

To measure neutrons of higher energy, an energy degrader is intended to be added right after the converter to slow down the recoil protons. This would allow the Recoil Proton Telescope to measure neutrons up to 70 or even $200 \mathrm{MeV}$. This requires an extensive work of optimization of the parameters of this new Telescope, namely the converter thickness as well as the degraders material and thicknesses.

\section{REFERENCES}

[1] H. Paganetti, Proton Therapy Physics. CRC Press, 2011

[2] ICRP, The 2007 Recommendations of the International Commission on Radiological Protection. ICRP Publication 103. Ann. ICRP 37 (2-4), 2007.

[3] S. B. Jia, M. H. Hadizadeha, A. A. Mowlavib and M. E. Loushab, Evaluation of energy deposition and secondary particle production in proton therapy of brain using a slab head phantom. Reports of Practical Oncology \& Radiotherapy Volume 19, Issue 6, November-December 2014, Pages 376-384

[4] M. R. Islam, Y. Zheng, T. L. Collums, J. M. Monson, S. Ahmad and E. R. Benton, Measurement ans simulation of secondary neutrons from uniform scanning proton beams in proton therapy. Radiation Measurements 96 (2017) 8-18

[5] Z. Riazi, H. Afarideh and R. Sadighi-Bonabi, Influence of ridge filter material on the beam efficiency and secondary neutron production in a proton therapy system. Z. Med. Phys. 22 (2012) 231-240

[6] Z. Vykydala, M. Králíka, J. Šolca, J. Vilímovskýb and V. Vondráčekb, Angular distribution of neutron spectral fluence around phantom irradiated with high energy protons. Radiation Measurements, Volume 92, September 2016, Pages 1-7
[7] X. Yan, U. Titt, A. M. Koehler and W. D. Newhauser, Measurement of neutron dose equivalent to proton therapy patients outside of the proton radiation field. Nuclear Instruments and Methods in Physics Research A 476 (2002) 429-434

[8] Y. C. Lin, C. C. Lee, T. C. Chao and H. Y. Tsai, Ambient neutron dose equivalent during proton therapy using wobbling scanning system : Measurements and calculations. Radiation Physics and Chemistry (2017)

[9] S. Trinkl, V. Mares, F. S. Englbrecht, J. J. Wilkens, M. Wielunski, K. Parodi, W. Rühm and M. Hillbrand, Systematic Out-of-field Secondary Neutron Spectrometry and Dosimetry in Pencil Beam Scanning Proton therapy. Med. Phys. 44 (5), May 2017

[10] M. R. Islam, T. L. Collums, Y. Zheng, J. Monson and E. R. Benton, Offaxis dose equivalent due to secondary neutrons from uniform scanning proton beams during proton radiotherapy. Phys. Med. Biol. 58 (2013) 8235-8251

[11] M. R. Islam, Off-axis Neutron Study from a Uniform Scanning Proton Beam Using Monte Carlo Code FLUKA. Master thesis, Oklahoma State University, 2013

[12] H. Jiang, B. Wang, X. G. Xu, H. D. Suit and H. Paganetti, Simulation of organ-specific patient effective dose due to secondary neutrons in proton radiation treatment. Phys. Med. Biol. 50 (2005) 4337-4353

[13] J. Farah, F. Martinetti, R. Sayah, V. Lacoste, L. Donadille, F. Trompier, C. Nauraye, L. De Marzi, I. Vabre, S. Delacroix, Monte Carlo modeling of proton therapy installations: a global experimental method to validate secondary neutron dose calculations. Physics in Medicine and Biology, Volume 59, Number 11 (2014) 2747-2765

[14] S. M. Valle, G. Battistoni, V. Patera, D. Pinci, A. Sarti, A. Sciubba, E. Spiriti and M. Marafini, The MONDO Project: A secondary neutron tracker detector for particle therapy. Nuclear Instruments and Methods in Physics Research A (2016)

[15] M. Marafini, L. Gasparini, R. Mirabelli, D. Pinci, V. Patera, A. Sciubba, E. Spiriti, D. Stoppa, G. Traini and A. Sarti, MONDO: a neutron tracker for particle therapy secondary emission characterisation. Phys. Med. Biol. 62 (2017) 3299-3312

[16] T. J. Langford, E. J. Beise, H. Breuer, C. R. Heimbach, G. Ji and J. S. Nico, Development and characterization of a high sensitivity segmented Fast Neutron Spectrometer (FaNS-2). Journal of Instrumentation, Volume 11, January 2016

[17] Nuclear Instruments and Methods in Physics Research A 506 (2003) 250-303. IEEE Transactions on Nuclear Science 53 No. 1 (2006) 270278. Nuclear Instruments and Methods in Physics Research A 835 (2016) 186-225.

[18] M. Kachel, D. Husson, S. Higueret, J. Taforeau and L. Lebreton, FastPixN, a new integrated pixel chip for a future fast version of the IRSN - Recoil Proton Telescope. Radiat Prot Dosimetry 214 Oct.; 161 (1-4):249-52.

[19] N. Arbor, S. Higueret, H. Elazhar, R. Combe, P. Meyer, N. Dehaynin, F. Taupin, D. Husson, Real-time detection of fast and thermal neutrons in radiotherapy with CMOS sensors. Phys Med Biol. 2017 Mar 7;62(5):1920-1934.

[20] N. Soppera, M. Bossant, E. Dupont, JANIS 4: An Improved Version of the NEA Java-based Nuclear Data Information System. Nuclear Data Sheets, Volume 120, June 2014, Pages 294-296. 